\title{
Progressive metastatic pulmonary calcification after successful renal transplantation
}

Metastatic pulmonary calcification is a complication of patients with renal failure [1], but is rarely seen after successful renal transplantation. A 51-year-old Japanese male demonstrated progression of diffuse pulmonary ground-glass attenuations over time. He had been treated with oral corticosteroids and immunosuppressants since undergoing left renal transplantation from his mother at 26 years of age. A chest radiograph (fig. 1a and b) and high-resolution computed tomography (fig. 1c, d, e, g and h) showed diffuse centrilobular groundglass attenuations and partly high-density areas. These findings had slowly worsened over time. ${ }^{99 \mathrm{~m}}$ Tc-methylene diphosphonate $\left({ }^{99 \mathrm{~m}} \mathrm{Tc}-\mathrm{MDP}\right)$ scintigraphy demonstrated high bilateral pulmonary uptake, especially in the upper and posterior lung fields (fig. 1f). This case is considered as continuously progressive metastatic pulmonary calcification after successful renal transplantation.

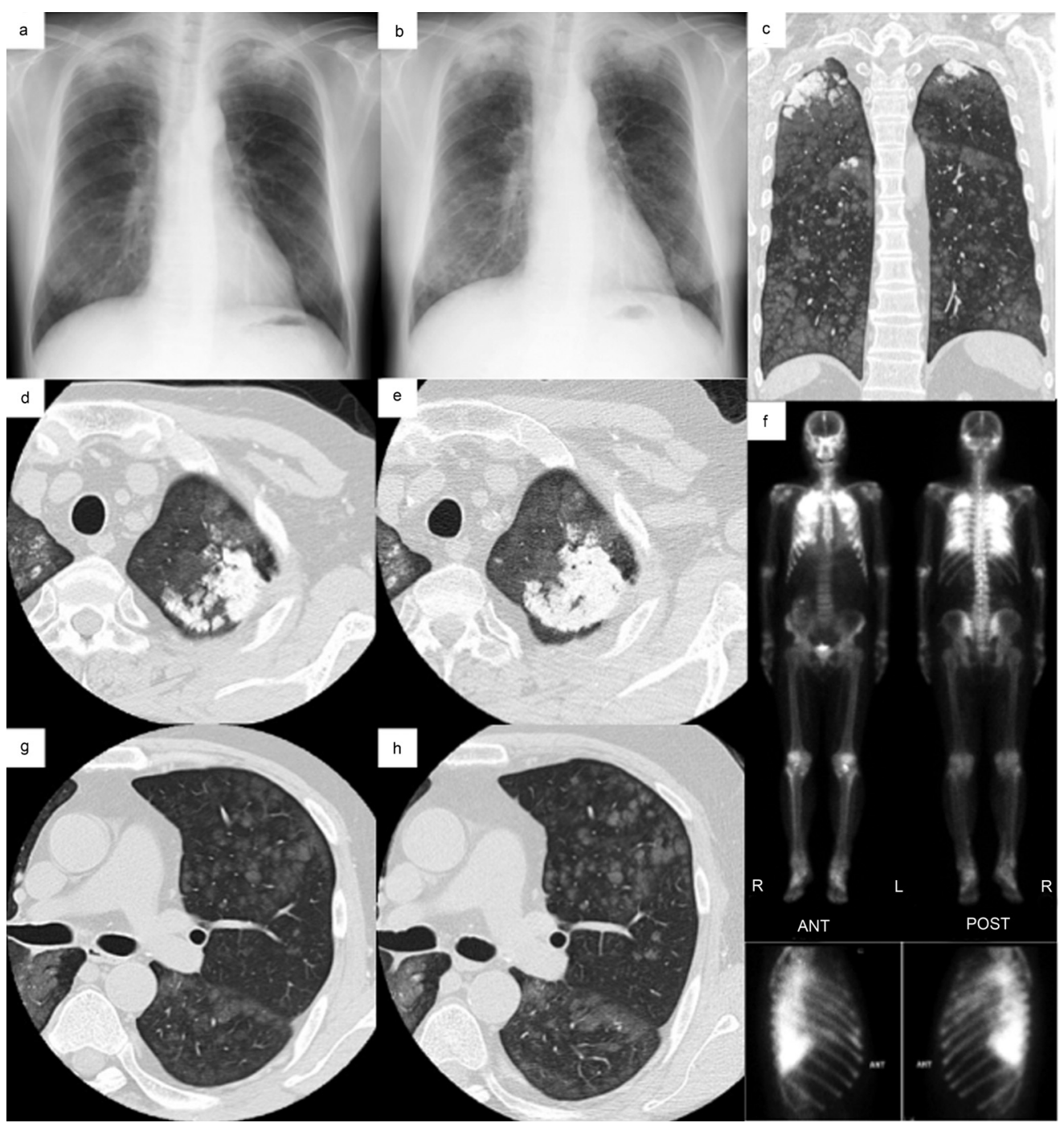

FIGURE 1. A chest radiograph from a) January 2003 and b) January 2012, and a computed tomography scan from d, g) December 2005 and c, e, h) November 2011 showing calcified nodular lesions and centrilobular ground-glass attenuations in the bilateral lungs. f) ${ }^{99 m}$ Tc-methylene diphosphonate scintigraphy from November 2011 high uptake of the agent was predominantly observed in the upper lung field and back area. 
To date, the comprehensive mechanism of metastatic pulmonary calcification is unknown, but a deposition of a calcium magnesium phosphate complex accumulating in the pulmonary interstitium and bronchovascular tissues is thought to be one mechanism [1]. Additionally, radiological findings of this disease on high-resolution computed tomography mimic other respiratory diseases such as hypersensitivity pneumonia and alveolar microlithiasis; ${ }^{99 \mathrm{~m}} \mathrm{Tc}-\mathrm{MDP}$ would be helpful in the diagnosis of early pulmonary calcifications [2].

\section{Kazuhiro Yatera, Toshinori Kawanami, Hiroshi Ishimoto and Hiroshi Mukae}

Dept of Respiratory Medicine, University of Occupational and Environmental Health, Japan, Fukuoka, Japan.

Correspondence: K. Yatera, Dept of Respiratory Medicine, University of Occupational and Environmental Health, Japan,
1-1 Iseigaoka, Yahata-nishi-ku, Kitakyushu, Fukuoka 807-8555, Japan. E-mail: yatera@med.uoeh-u.ac.jp

Statement of Interest: None declared.

Provenance: Submitted article, peer reviewed.

\section{REFERENCES}

1 Chan ED, Morales DV, Welsh CH, et al. Calcium deposition with or without bone formation in the lung. Am J Respir Crit Care Med 2002; 165: 1654-1669.

2 Brodeur FJ, Kazerooni EA. Metastatic pulmonary calcification mimicking air-space disease: technetium-99m-MDP SPECT imaging. Chest 1994; 106: 620-622. 